\title{
In situ measurements of sub-meter plasma waves over low-latitude ionosphere during Leonid-99 meteor storm
}

\author{
S. P. Gupta, R. Sekar, and Y. B. Acharya \\ Physical Research Laboratory, Ahmedabad 380 009, India \\ Received: 10 October 2003 - Revised: 25 February 2004 - Accepted: 8 March 2004 - Published: 14 June 2004
}

\begin{abstract}
In situ probe measurements of plasma parameters were carried out on 18 and 20 November 1999 from Sriharikota, India, a low-latitude rocket launching station to investigate the effect of a Leonid meteor storm. Results obtained on plasma waves using a high frequency Langmuir probe are discussed. The characteristics of the sub-meter scale size plasma waves observed for the first time during Leonid meteor storm are presented. Based on the results obtained from both the rocket flights and comparison with the results obtained from previous rocket flights from the same location, it appears these sub-meter waves are associated with intense meteoric activity. A possible mechanism based on the dependence of the meteoric activity and its limitations are discussed.
\end{abstract}

Key words. Ionosphere (Equatorial ionosphere; Ionospheric irregularities; Plasma waves and instabilities)

\section{Introduction}

It is known that a typical meteor Zenith Hourly Rate (ZHR) of a Leonid meteor shower associated with Comet TempelTuttle, which occurs every year during $17-18$ November, is around 5 (McKinley, 1961). However, the ZHR for a Leonid meteor storm, an event which occurs once in 33 years, is about a few thousands (Aguirre, 2000). The meteor streams enter the Earth's ionosphere with the velocities ranging from $12 \mathrm{~km} / \mathrm{s}$ to $72 \mathrm{~km} / \mathrm{s}$ (Hocking, 2000). It is well established that the meteoric species continuously become deposited in the lower thermosphere both in the form of ions due to ablation of the meteorites and also in the form of neutral species. A few in situ measurements (Narcisi, 1968; Shirke et al., 1981) during normal shower activity are available in the literature. The in situ measurement during an intense meteor storm is not available in the literature (McNeil et al., 2001), particularly from a low-latitude region. However, rocket experiments were conducted during a Leonid storm in 1999

Correspondence to: R. Sekar

(rsekar@prl.ernet.in from Sriharikota (SHAR), India, with an objective to investigate the effects on plasma parameters and the possible plasma waves in the low-latitude ionosphere induced by intense meteor storm. Based on a single rocket flight, the preliminary result on the existence of a new type of plasma wave over low latitude during a Leonid meteor storm is reported elsewhere (Gupta et al., 2003). The present communication deals with the detailed results obtained from two rocket flights on plasma waves and brings out their association with Leonid meteor storm activity.

The plasma waves in equatorial $\mathrm{E}$ region associated with equatorial electrojet have been studied using in situ probes (Prakash et al., 1972; Pfaff et al., 1987) and VHF radars (Balsley and Farley, 1971; Prakash et al., 1974). VHF radars provide temporal behaviour of plasma waves at a particular wavelength, while the in situ probe measurements provide information of various ranges of scale sizes during a limited duration. The earlier studies based on these techniques revealed that there exists two types of plasma waves (conventional two stream and gradient drift waves) over the equatorial ionosphere. As the site (dip lat $6^{\circ} \mathrm{N}$ ) of the observation reported in the present communication is far from the equatorial electrojet effects, the conventional two stream waves are unlikely to become excited. As it can be noticed from the following section that the presence of the observed plasma waves in the present case in a shallow plasma density gradient region and the absence of the same in a steep plasma density gradient region rule out the possibility of gradient drift waves. Further, the scale sizes of the observed plasma waves are considerably lesser than the cut-off wavelength of conventional plasma waves established by the earlier works (Balsley and Farley, 1971; Schmidt and Gary, 1973; Pfaff et al., 1987). Hence the conventional two steam and gradient drift processes cannot account for the new plasma waves. Thus, these conventional processes will not be discussed any further.

In the present communication, the characteristic of a new plasma wave, its association with meteor storm activity and a possible mechanism with its limitation will be discussed. 

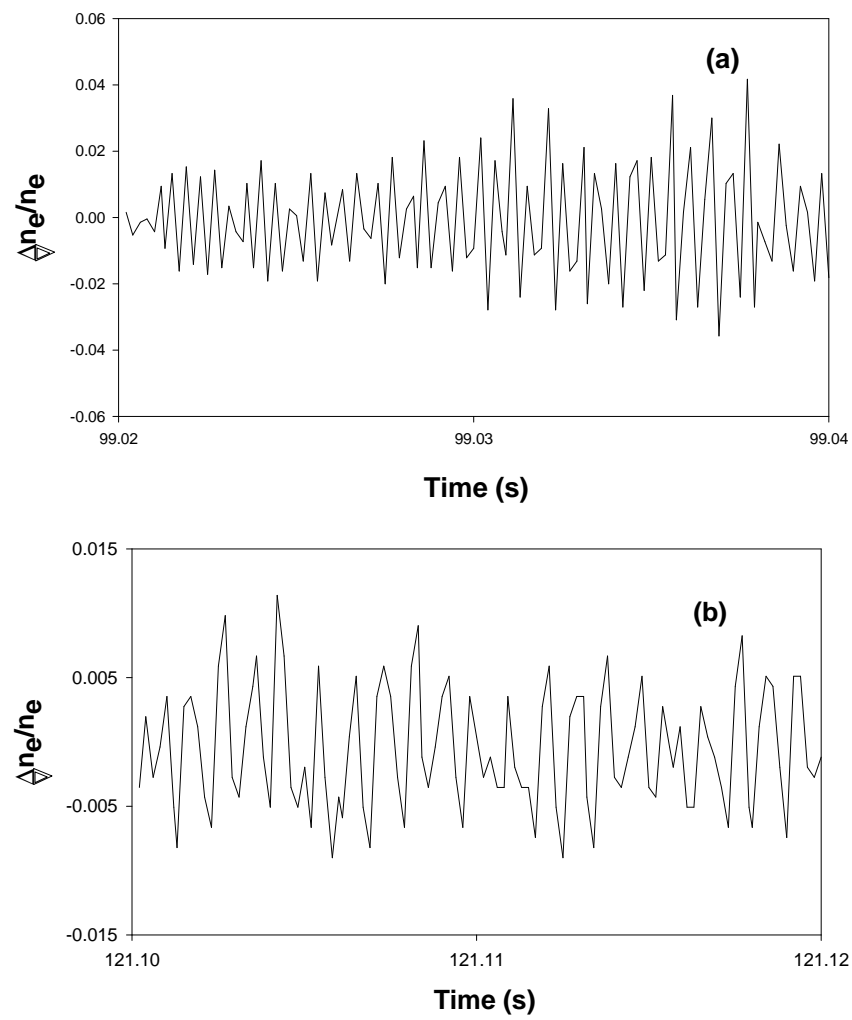

Fig. 1. Telemetry record of plasma waves obtained on 18 November 1999 , (a) at an altitude of $105 \mathrm{~km}$ and (b) at $120 \mathrm{~km}$ altitude.

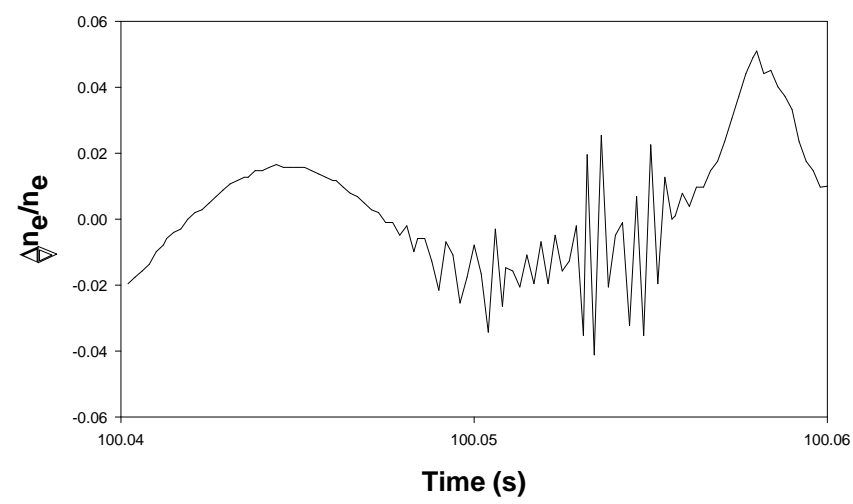

Fig. 2. Telemetry record of the plasma waves obtained at $105 \mathrm{~km}$ altitude on 20 November 1999 when the meteor activity level is one-third in comparison to the first flight.

\section{Details of the experiments}

Two RH-300 Mark II rockets (F01 and F02) with identical instruments were launched from SHAR $\left(13.7^{\circ} \mathrm{N}, 80.2^{\circ} \mathrm{E}\right.$, dip lat. $6.0^{\circ} \mathrm{N}$ ), on 18 and 20 November 1999 , respectively, at 7:25 and 7:03 IST (Indian standard time). Both the rockets were launched nearly with the same launch azimuth of $90^{\circ}$ and a launch elevation of $84^{\circ}$. The rockets attained apogee of 139 and $136 \mathrm{~km}$, respectively. The rocket carried a high frequency Langmuir probe and an electric field probe to mea- sure plasma wave parameters. The electric field probe did not yield any useful scientific information as the rocket velocity induced electric field could not be eliminated satisfactorily. The uncertainty in the measurement of the plasma wave amplitude is less than $0.4 \%$ of the ambient plasma density for the present case.

\section{Results}

Figure 1a depicts the telemetry record of high frequency $(100 \mathrm{~Hz}$ to $3 \mathrm{kHz})$ channel data of the normalized fluctuations in electron densities during $99.02 \mathrm{~s}$ to $99.04 \mathrm{~s}$, corresponding to a $105 \mathrm{~km}$ altitude. The normalized amplitude of fluctuation (peak to peak) at this altitude corresponds to about $4 \%$ of the ambient electron density. During the time interval of $0.02 \mathrm{~s}$, around forty-two peaks are observed which correspond to the frequency of $2.1 \mathrm{kHz}$ in the rocket frame of reference when the rocket velocity is $1 \mathrm{~km} / \mathrm{s}$. The amplitude and the wave frequency in the rocket frame of reference of these plasma waves are found to change with altitudes. Thus, in order to ascertain that the observed plasma waves are geophysical in nature, sample data at a different altitude is analyzed. Figure $1 b$ depicts a telemetry record of high frequency oscillations in the same channel $(100 \mathrm{~Hz}$ to $3 \mathrm{kHz})$ but at a different altitude region where the vertical component of rocket velocity is only $0.5 \mathrm{~km} / \mathrm{s}$. The peak-to-peak amplitude of the wave reduced to nearly $1 \%$ of the ambient. During a time of interval of $0.02 \mathrm{~s}$, around 25 peaks are observed which correspond to a $1.25 \mathrm{kHz}$ wave frequency in the rocket frame of reference.

The telemetry record of the high frequency channel obtained from flight F02 between 100.04 to $100.06 \mathrm{~s}$, corresponding to $105 \mathrm{~km}$ altitude, is depicted in Fig. 2. High frequency fluctuations are observed at $100.05 \mathrm{~s}$ with a normalized amplitude equal to $3.5 \%$ of the ambient electron density. During a time interval of $0.008 \mathrm{~s}$, seventeen peaks are seen which correspond to a $\sim 2.1 \mathrm{kHz}$ wave frequency in the rocket frame of reference when the rocket velocity is $1 \mathrm{~km} / \mathrm{s}$. Figure 2 represents a sample of high frequency waves in plasma density observed by the second rocket, which was conducted when the Leonid storm activity level had fallen to one-third of its value corresponding to 18 November.

Figures $3 \mathrm{a}$ and $\mathrm{b}$ depict the altitude profiles of the amplitudes of the waves, along with electron density profiles obtained on 18 and 20 November, respectively. The plasma waves are observed to confine in the altitude region of 100 to $120 \mathrm{~km}$ on 18 November and between 100 and $106 \mathrm{~km}$ on 20 November. The plasma wave amplitude attains a maximum at $105 \mathrm{~km}$ on 20 November and the wave amplitude is below the detection limit beyond $106 \mathrm{~km}$. The altitude extent varies with the level of Leonid storm activity. The maximum amplitude is found to be nearly at the same altitude $(105 \mathrm{~km})$ on both days. The fluctuations observed near the rocket apogee are non-geophysical in nature.

In general, the electron density profile is much more structured on 18 November compared to the profile on 
20 November. Further, a small variation in electron density profile is noticed on 18 November and a relatively significant one on 20 November, closer to the altitude region where plasma wave amplitude is significant. A sharp cut-off in the amplitude of the plasma wave is seen on 20 November in Fig $3 b$ when the Leonid storm activity is less.

\section{Discussion}

Figure 1a and $\mathrm{b}$ reveals that the electron density fluctuations are not only different in amplitudes but also different in dominant frequency in the rocket frame of reference. The differences in the frequency at different altitudes suggest that these fluctuations are not associated with a measurement system. Taking into account the measured velocities $(\mathrm{V})$ of the rocket at different altitudes, and the observed dominant frequencies (f), the scale sizes of waves (V/f) are calculated to be $47 \mathrm{~cm}$ and $40 \mathrm{~cm}$, respectively, at 105 and $120 \mathrm{~km}$ altitudes. Thus, these scale sizes of the plasma wave fall under the sub-meter category. Further, Fourier spectrum analysis of these plasma waves (not shown in the figure) also reveals that the power is centered at the region of sub-meter scale size.

In this context, it is important to study the association between the sub-meter plasma waves and the activity of the Leonid meteor storm. Since the activity on 20 November had fallen to about one-third of its value corresponding to 18 November, the information regarding the presence (or absence) of the plasma waves on a non-meteor storm day could not be obtained by the present set of measurements. However, the earlier flights conducted from the same location on non-meteor storm days with identical probe systems did not reveal the presence of sub-meter category of plasma waves (Thiemann et al., 1995; Gupta, 1997). Further, it is clear from the results (Figs. 3a and b) that the altitude extents of the region wherein the plasma waves are present depend on the activity of the Leonid storm. It is interesting to note that a maximum amplitude of plasma waves exist at an altitude $(105 \mathrm{~km})$ where the meteoric deposition is large in general. The recent measurement (Close et al., 2002) on the head echoes from the Leonid meteor shower which occurred during 1998, using UHF ALTAIR radar, supports this view. Further, the maximum amplitude of the sub-meter plasma waves at about $105 \mathrm{~km}$ is $4 \%$ with an amplitude that is four times more than the usual plasma waves. These experimental evidences indicate that the sub-meter waves are associated with Leonid storm activity.

Regarding the generation of the sub-meter plasma waves, the recent simulation presented by Dyrud et al. (2002) becomes important. In order to explain non-specular VHF and UHF radar echoes from meteor trails observed over the low-latitude region, (Kwajelein Atol, a place having a dip value of the Earth's magnetic field, similar to the present launching station, SHAR) kinetic plasma simulations within a meteor trail have been carried out by Dyrud et al. (2002). These simulations reveal that the meteor trail often develops Farley-Buneman gradient drift waves which become tur-
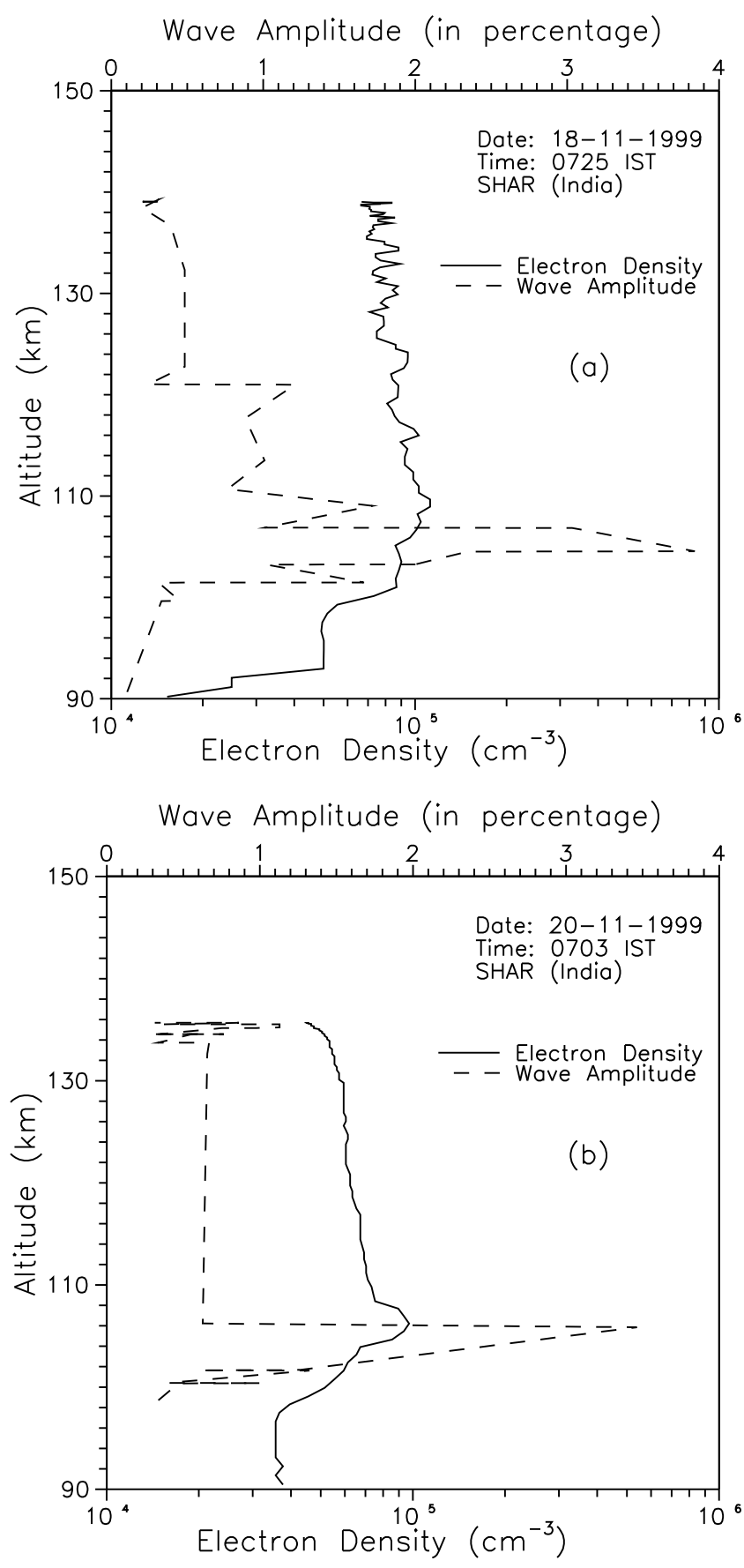

Fig. 3. Altitude profiles of amplitude of plasma waves along with electron densities obtained (a) on 18 November 1999, (b) on 20 November 1999.

bulent and generate field-aligned irregularities of sub-meter scale sizes in an altitude region $96 \mathrm{~km}$ to $106 \mathrm{~km}$. The simulation and earlier computation work by Farley (1963) require that the magnitude of electron drift velocity perpendicular to the magnetic field should be $4 \mathrm{~km} / \mathrm{s}$ for the generation of sub-meter waves. Such high velocities do exist within the meteor trail. The observations, with changes in the altitude profile of the electron density but to a lesser 
extent on 18 November, and a relatively significant change on 20 November at $105 \mathrm{~km}$, where a maximum plasma wave amplitude is observed, support this mechanism of the generation of sub-meter waves. However, their simulation indicates that the actual altitude range for meteor trail instability depends on meteor trail velocity. In the present experimental conditions, the meteor trail velocity is expected to be on the higher side as the Leonid storm activity and the observation took place in the morning sector over the low-latitude region. The actual measurement (Close et al., 2002) of the average meteor trail speed of the Leonid meteor shower which occurred during 1998 when the condition was similar to the present case was $69 \mathrm{~km} / \mathrm{s}$. This would not favor the growth of sub-meter plasma waves at a higher altitude $(>106 \mathrm{~km})$ region owing to a larger radius of the meteor trail and a corresponding reduction in the electron density gradient within the meteor trail according to the simulation of Dyrud et al. (2002). However, the plasma waves in the present rocket campaign were observed in different parts of the rocket trajectory even at a $120 \mathrm{~km}$ altitude (see Fig. 1b). The presence of waves was also recorded during the down leg of the rocket trajectory. In the absence of the spatial distribution of the meteor trails in the ambient ionosphere during a severe Leonid meteor storm, the above mechanism appears to be a viable one. Further investigations are needed to understand the existence of plasma waves at a higher altitude region, particularly on a meteor storm day.

\section{Summary}

The characteristics of the sub-meter plasma waves over a low-latitude E-region observed for the first time during Leonid meteor storm of November 1999 are presented. Indications for the association of the observed sub-meter waves with the activity of Leonid meteor storm are found. A possible mechanism and its limitations are discussed.

Acknowledgements. We acknowledge the Payload Integration group at VSSC, Thiruvananthapuram and the launch operation personnel at SHAR rocket range for conducting both the rocket flights successfully. We thank Director, PRL, for taking keen interest in this project. The work is supported by Department of Space, Government of India.

Topical Editor M. Lester thanks a referee for his help in evalutating this paper.

\section{References}

Aguirre, E. L.: Leonid storm on schedule, Sky \& Telescope, Feb. issue, 120, 2000.

Balsley, B. B. and Farley, D. T.: Radar studies of equatorial electrojet at three frequencies, J. Geophy. Res., 76, 8341-8351, 1971.

Close, S., Hunt, S. M., McKeen, F. M., and Minardi, M. J.: Characterization of Leonid meteor head echo data collected using the VHF-UHF Advanced Research Projects Agency Long-Range Tracking and Instrumentation Radar (ALTAIR), Radio Sci., 37, doi:10.1029/2000RS002602, 2002.

Dyrud, L. P., Oppenheim, M. M., Close, S., and Hunt, S.: Interpretation of non-specular radar meteor trails, Geophys. Res. Lett. 29 (21), 2012, doi:10.1029/2002GL015953, 2002.

Farley, D. T.: A plasma instability resulting in field aligned irregularities in the ionosphere, J. Geophy. Res., 68, 6083-6097, 1963.

Gupta, S. P.: Features of E region irregularities at the magnetic equator and in its vicinity, Adv. Space Res., 20, No. 11, 21952198, 1997.

Gupta, S. P., Sekar, R., and Acharya, Y. B.: A new plasma wave over low-latitude ionosphere during Leonid meteor storm, Current Science, 84, 1340-1342, 2003.

Hocking, W. K.: Real time meteor entrance speed determinations made with interferometric meteor radars, Radio Sci., 35, 12051220, 2000.

McKinley, D. W. R.: Meteor science and engineering, McGraw-Hill Book Co., New York, 146-147, 1961.

McNeil, W. J., Dressler, R. A., and Murad, E.: Impact of major meteor storm on Earth's ionosphere : A modeling study, J. Geophy. Res., 106, 10 447-10 465, 2001.

Narcisi, R. S.: Process associated with metal ion layers in the E region of ionosphere, Space Res., VIII, 360-369, 1968.

Pfaff, R. T., Kelley, M. C., Kudeki, E., Fejer, B. G., and Baker, K. D.: Electric field and plasma density measurements in the strongly driven day time equatorial electrojet, 2. two stream waves, J. Geophy. Res., 92, 13 597-13612, 1987.

Prakash S., Subbaraya, B. H., and Gupta, S. P.: Rocket measurements of ionization irregularities in the equatorial ionosphere at Thumba and identification of plasma irregularities, Indian J. Radio space Phy., 1, 72-80, 1972.

Prakash S., Jain, C. L., Balsley, B. B., and Greenwald, R. W.: Evidence of two types of electron density irregularities in the electrojet over Thumba, India, J. Geophs. Res., 79, 4334-4336, 1974.

Schmidt, M. J. and Gary, S. P.: Density gradients and FarleyBuneman instability, J. Geophy. Res., 78, 8261-8265, 1973.

Shirke, J. S., Sridharan, R., Gupta, S. P., Danilov, A. D., Pokhunkov, A. A., Semenov, V. K. and Verfolomeev, V. A.: On the formation of sharp layer of metallic constituents at sun rise in the lower thermosphere, Proc. Indian Acad. Sci., 91, 141-146, 1981.

Thiemann-Rhode, H. V., Piel, A., Steigies, C., and Gupta, S. P.: Gradient drift waves at the edge of equatorial electrojet over SHAR, Proc. 12th ESA Symp. On rockets and balloon programme, Lillehammer, Norway, 291-294, 1995. 